\title{
Hepatoprotective studies on methanolic extract fractions of Lindernia ciliata and development of qualitative analytical profile for the bioactive extract
}

\author{
Praneetha Pallerla* (D), Narsimha Reddy Yellu and Ravi Kumar Bobbala
}

\begin{abstract}
Background: The objective of the study is to evaluate the hepatoprotective activity of methanolic extract fractions of Lindernia ciliata (LC) and development of qualitative analytical profile of the bioactive fraction using HPLC fingerprinting analysis. All the fractions of methanolic extract of Lindernia ciliata (LCME) are assessed for their total phenolic, flavonoid contents and in vitro antioxidant properties by using DPPH, superoxide, nitric oxide, hydroxyl radical scavenging activities and reducing power assay. Acute toxicity study was conducted for all the fractions and the two test doses 50 and $100 \mathrm{mg} / \mathrm{kg}$ were selected for the hepatoprotective study. Liver damage was induced in different groups of rats by administering $3 \mathrm{~g} / \mathrm{kg}$.b.w.p.o. paracetamol and the effect of fractions were tested for hepatoprotective potential by evaluating serum biochemical parameters and histology of liver of rats. The effective fraction was evaluated for its antihepatotoxic activity against D-Galactosamine (400 mg/kg b.w. i.p.) and in vivo antioxidant parameters viz., Glutathione (GSH), Melondialdehyde (MDA) and Catalase (CAT) levels are estimated using liver homogenate.

Results: Among all the fractions, butanone fraction of LCME, (BNF-LCME) has shown better hepatoprotective activity and hence it is selected to evaluate the antihepatotoxicity against D-GaIN. The activity of BNF-LCME is well supported in in vitro and in vivo antioxidant studies and may be attributed to flavonoidal, phenolic compounds present in the fraction. Hence, BNF-LCME was subjected to the development of qualitative analytical profile using HPLC finger printing analysis.
\end{abstract}

Conclusions: All the fractions of LCME exhibited significant hepatoprotective activity and BNF-LCME $(50 \mathrm{mg} / \mathrm{kg})$ was identified as the most effective fraction.

Keywords: Antioxidants, D-galactosamine, Lindernia ciliata, Hepatoprotective activity, HPLC

\section{Background}

Lindernia ciliata (Colsm.) Pennell of family Linderniaceae is a low growing, stoloniferous, mat-forming, annual, herb from $0.1-0.2 \mathrm{~m}$ high. It is restricted to the tropics and sub-tropics of Asia, northern Australia and North America. In India it is found as an insignificant weed, mainly in rice fields [1]. Traditionally it is used as a remedy for urinary disturbances, gonorrhea, headache, spleen diseases, asthma, jaundice, bronchitis, liver

\footnotetext{
* Correspondence: praneetha.sruthi@gmail.com

University College of Pharmaceutical Sciences, Kakatiya University, Warangal, Telangana state 506009 , India
}

complaints, constipation, fever, loss of appetite, cough and skin diseases [2]. In view of its traditional medicinal use in the treatment of liver disorders, the present study was designed to separate the methanolic extract fractions of Lindernia ciliata and evaluate their hepatoprotective activity against three mechanistically devised models viz. paracetamol, ethanol and D-galactosamine induced hepatotoxicity in rats and its in vitro antioxidant activity. Furthermore, the qualitative analytical profile is developed for the bioactive fraction using HPLC fingerprinting analysis. 


\section{Materials and methods \\ Animals}

Wistar albino rats weighing 150-200 g were purchased from Sainath agencies, Hyderabad, Telangana, India with a prior permission from our institutional animal ethical committee (1820/GO/Re/S/15/CPCSEA, Date:01-092015) and used for the studies. The animals were caged under constant environmental and nutritional conditions (12:12 h light and dark cycle; at an ambient temperature of $25 \pm 5^{\circ} \mathrm{C}$; $35-60 \%$ of relative humidity). They had free access to food and water ad libutum.

\section{Collection and preparation of extracts}

The whole plants of Lindernia ciliata were collected in August 2015, from cotton fields of Bhayyaram, Warangal, Telangana state, India. The plant was authenticated by Prof. V.S. Raju, taxonomist, Kakatiya University, Warangal. A voucher specimen of the plant (KU/UCPSC/54) is being maintained in the herbarium of Department of Pharmacognosy and Phytochemistry, University College of Pharmaceutical Sciences, Kakatiya University, Warangal.

The whole plants were dried thoroughly under shade, powdered coarsely and macerated with methanol in a round bottomed flask for 7 days with stirring at regular intervals and filtered after 7 days. It is then concentrated under reduced pressure using Rotavapour evaporator, (Buchi Rotavapour, Switzerland) to yield a semisolid mass (7.2\%) and coded as LCME. A Pilot study was conducted on LCME to fractionate it with toluene, ethyl acetate, butanone and n-butyl alcohol in succession. Based on the yield and TLC profile of the fractions, the solvents- toluene and butanone were selected for fractionation of LCME.

$60 \mathrm{~g}$ of LCME was suspended in $500 \mathrm{~mL}$ of water and fractionated with toluene and butanone in succession. The obtained fractions were concentrated under reduced pressure to yield corresponding extracts. They were given the codes, as TLF-LCME (Toluene fraction), BNFLCME (butanone fraction) and AQF-LCME (Aqueous fraction- the residue left in the water after fractionation process).

\section{Total phenolic content}

The total phenolic content of the fractions of LCME was determined using the Folin-Ciocalteu colorimetric method [3]. The total phenolic content was expressed as Gallic acid equivalents (GAE) in mg per gram of extract.

\section{Total flavonoid content}

The total flavonoid content of the fractions of LCME was measured using the aluminium chloride colorimetric method [3]. It was expressed as Rutin equivalents (RE) in $\mathrm{mg}$ per gram of extract.

\section{In vitro antioxidant studies}

The fractions of LCME were screened to assess their antioxidant property by DPPH radical scavenging assay [4], superoxide scavenging activity [5], nitric oxide scavenging activity [6], hydroxyl radical scavenging activity [7], and reducing power assay [8].

\section{Acute toxicity study}

Acute toxicity study was carried out for all the fractions, TLF-LCME, BNF-LCME and AQF-LCME according to the OECD 423 guidelines [9] using female Wistar albino rats. They were observed for signs of toxicity and mortality for $72 \mathrm{~h}$.

\section{Assessment of hepatoprotective activity of fractions of LCME against paracetamol induced hepatotoxicity}

The rats were divided into nine groups of six each, control, toxic, standard, and six test groups. The procedure was followed from Sabeena and Ajay, [10] with minor modifications.

Group I (Control group): Treated with vehicle, $(1 \mathrm{ml} /$ $\mathrm{kg}$ b.w. of $2 \%$ gum acacia in water) daily for 7 days.

Group II (Toxic group): Treated with vehicle $(1 \mathrm{ml} / \mathrm{kg}$ b.w of $2 \%$ gum acacia in water) daily for 7 days followed by paracetamol on the eighth day.

Group III (Silymarin $100 \mathrm{mg} / \mathrm{kg}$ ), Group IV (TLF-LCME $50 \mathrm{mg} / \mathrm{kg}$ ), Group V (TLF-LCME $100 \mathrm{mg} / \mathrm{kg}$ ), Group VI (BNF-LCME $50 \mathrm{mg} / \mathrm{kg}$ ), Group VII (BNF-LCME $100 \mathrm{mg} /$ $\mathrm{kg}$ ), Group VIII (AQF-LCME $50 \mathrm{mg} / \mathrm{kg}$ ) and Group IX (AQF-LCME $100 \mathrm{mg} / \mathrm{kg}$ ) were treated with their respective standard or extracts followed by paracetamol on eighth day.

The blood and liver samples were collected from the animals of all groups $24 \mathrm{~h}$ after administration of paracetamol, for estimation of various serum biochemical parameters and histological studies respectively.

\section{Assessment of antihepatotoxic activity of bioactive fraction against D-Galactosamine induced hepatotoxicity in rats}

It was done according to Karan et al., [11] The rats were divided into five groups of six animals each. Group I served as normal and is given the vehicle i.e., $2 \%$ gum acacia in water $(1 \mathrm{~mL} / \mathrm{kg}$ b.w p.o) for 3 days. Toxic group (Group II), standard (Group III) and two test groups (Group IV, V) were given a single dose of DGalactosamine $(400 \mathrm{mg} / \mathrm{kg}$ intra peritoneal) on day 1 followed by vehicle ( $2 \%$ gum acacia $1 \mathrm{ml} / \mathrm{kg}$ b.w.p.o.), Silymarin (100 mg/kg b.w), BNF-LCME (50 mg/kg b.w) and BNF-LCME (100 mg/kg b.w) respectively for three times at the time point of $2 \mathrm{~h}, 24 \mathrm{~h}, 48 \mathrm{~h}$ after the administration of D-Galactosamine. The blood and liver samples were collected from the animals $1 \mathrm{~h}$ after the last treatment for determination of various serum biochemical 
parameters, in vivo antioxidant parameters and histological studies respectively.

\section{Histological studies}

The livers from all the animals were isolated and fixed in formalin solution and processed for histopathological examination.

\section{Determination of prothrombin time (PT)}

The prothrombin time was determined [12] by collecting blood from animals in normal capillary tubes and the capillaries were broken down into pieces until a thread was observed, and the clotting time was noted.

\section{Development of qualitative analytical profile for the bioactive fraction \\ High performance liquid chromatography}

The distinct chemo profile of the bioactive fraction was developed using HPLC. Shimadzu UFLC unit connected to Photo Diode Array detector was used. The column used was obtained from Grace smart $(250 \mathrm{~mm} \times 4.5 \mathrm{~mm}$, Reverse phase C-8, $5 \mu \mathrm{m}$ particle size). The details of the parameters maintained for the HPLC analysis are Mobile Phase: Methanol: Water: 10:90 (V/V); sample concentration: $100 \mu \mathrm{g} / \mathrm{ml}$; volume of sample applied: $20 \mu \mathrm{l}$ at detection wavelength: $220 \mathrm{~nm}$.

\section{Statistical analysis}

The data obtained were analyzed by one-way ANOVA followed by Dunnett's multiple comparisons test using GraphPad Prism version 3 (GraphPad Software, La Jolla California USA)

\section{Results and discussion}

\section{Total phenolic and flavonoid contents}

The phenolic and flavonoid contents of the fractions of LCME are shown in Table 1. Among all the fractions BNF-LCME has shown more phenolic and flavonoid contents i.e., $121.76 \mathrm{mg}$ of $\mathrm{GAE} / \mathrm{g}$ of extract and 34.48 $\mathrm{mg}$ of RE/g of extract respectively.

Table 1 The total phenolic and flavonoid contents of the fractions of LCME

\begin{tabular}{lll}
\hline Groups & $\begin{array}{l}\text { Total phenolic content } \\
\text { (mg of GAE/g of extract) }\end{array}$ & $\begin{array}{l}\text { Total flavonoid content } \\
\text { (mg of RE/g of extract) }\end{array}$ \\
\hline TLF- LCME & $51.79 \pm 1.11$ & - \\
BNF-LCME & $394.31 \pm 0.44$ & $115.09 \pm 0.32$ \\
AQF -LCME & $96.25 \pm 0.29$ & $30.12 \pm 0.48$ \\
\hline
\end{tabular}

Data expressed as mean \pm SD, $n=3$

GAE gallic acid equivalents, RE rutin equivalents, TLF-LCME Toulene fraction of methanolic extract fraction of Lindernia ciliata, BNF-LCME Butanone fraction of methanolic extract fraction of Lindernia ciliata, AQF-LCME Aqueous fraction of methanolic extract fraction of Lindernia ciliata

\section{In vitro antioxidant studies}

All the three fractions of LCME have shown a concentration dependent in vitro free radical scavenging activity. The $\mathrm{IC}_{50}$ of fractions of LCME with their corresponding standards are shown in Table 2.

\section{Reducing power assay}

The results are expressed in terms of ascorbic acid equivalents (AAE). The reducing power of TLF-LCME, BNF-LCME and AQF-LCME were found to be $4.16 \pm$ $0.25,18.34 \pm 0.27$ and $6.59 \pm 0.95 \mathrm{mg}$ of AAE/g of extract respectively. Among all the fractions BNF-LCME has shown better activity which may be attributed due to the presence of more phenolic and flavonoid contents.

\section{Acute toxicity study}

The various fractions of LCME were found to be non toxic up to a dose of $1000 \mathrm{mg} / \mathrm{kg}$ in albino rats. Since LD50 of the fractions was greater than $1000 \mathrm{mg} / \mathrm{kg}$ b.w., the investigations on these fractions were carried out with two graded doses i.e. 50 and $100 \mathrm{mg} / \mathrm{kg}$ b.w.

\section{Assessment of hepatoprotective activity of fractions of LCME against paracetamol induced hepatotoxicity}

The rats treated with overdose of paracetamol $(3 \mathrm{~g} / \mathrm{kg})$ caused significant liver damage which was evident from the changes in serum biochemical parameters and histology of liver of rats. The results are shown in Table 3 and histological results are shown in Fig. 1. The level of hepatospecific enzymes such as [Aspartate aminotransferase (AST), Alanine aminotransferase (ALT), Alkaline phosphatase (ALP)], Lactate dehydrogenase (LDH) and Gamma glutamyl transferase (GGT) were increased in serum indicating damage to the liver cell plasma membrane as these enzymes are normally present in cytoplasm. Pretreatment with all the three fractions of LCME at 50 and $100 \mathrm{mg} / \mathrm{kg}$ significantly reduced the elevated level of these enzymes in paracetamol treated rats. This reflects the ability of the fractions in preserving the structural integrity and protection of hepatocellular membrane which is attributed to their antioxidant activity. Paracetamol produces sufficient injury to hepatic parenchyma to cause large increase in bilirubin content [13]. Also a decrease in TP, albumin and prothrombin occurs due to the disruption and dissociation of polyribosome on endoplasmic reticulum leading to decrease in protein biosynthesis. Pretreatment with fractions of LCME at both test doses significantly $(p<0.01)$ decreased the bilirubin level, prothrombin time and increased the level of TP and ALB indicating the ability of the extracts in preventing the injury to hepatic parenchyma and facilitating the uninterrupted protein biosynthesis respectively. The histopathological examination of liver 
Table 2 In vitro antioxidant activity of fractions of LCME and their corresponding standards

\begin{tabular}{|c|c|c|c|c|}
\hline Method & $\begin{array}{l}\text { IC50 value of the } \\
\text { extract TLF-LCME } \\
\text { in } \mu \mathrm{g} / \mathrm{mL}\end{array}$ & $\begin{array}{l}\text { IC50 value of the } \\
\text { extract BNF-LCME } \\
\text { in } \mu \mathrm{g} / \mathrm{mL}\end{array}$ & $\begin{array}{l}\text { IC50 value of the } \\
\text { extract AQF-LCME } \\
\text { in } \mu \mathrm{g} / \mathrm{mL}\end{array}$ & $\begin{array}{l}\text { IC50 value of the } \\
\text { standard in } \mu \mathrm{g} / \mathrm{mL}\end{array}$ \\
\hline DPPH & $95.7 \pm 3.2$ & $25.5 \pm 2.6$ & $39.3 \pm 1.6$ & $\begin{array}{l}0.67 \pm 0.52 \\
\text { (Rutin) }\end{array}$ \\
\hline Nitric Oxide & $65.23 \pm 5.5$ & $31.69 \pm 1.8$ & $61.31 \pm 2.8$ & $\begin{array}{l}5.16 \pm 0.24 \\
\text { (Ascorbic acid) }\end{array}$ \\
\hline Superoxide & $623.4 \pm 4.9$ & $214.6 \pm 6.7$ & $421.11 \pm 4.5$ & $\begin{array}{l}4.16 \pm 0.05 \\
\text { (Rutin) }\end{array}$ \\
\hline Hydroxyl radical & $524.9 \pm 3.2$ & $102.18 \pm 6.1$ & $195.17 \pm 6.1$ & $\begin{array}{l}6.57 \pm 0.18 \\
\text { (Mannitol) }\end{array}$ \\
\hline
\end{tabular}

Data expressed as mean $\pm \mathrm{SD}, n=3$

TLF-LCME Toulene fraction of methanolic extract fraction of Lindernia ciliata, BNF-LCME Butanol fraction of methanolic extract fraction of Lindernia ciliata, AQF-LCME Aqueous fraction of methanolic extract fraction of Lindernia ciliata

of rats treated with paracetamol showed centrilobular necrosis, inflammatory infiltration of lymphocytes, congestion of sinusoidal spaces, and bleeding in hepatic lobes. Pretreatment with fractions and Standard (Silymarin $100 \mathrm{mg} / \mathrm{kg})$ exhibited a significant $(P<0.001)$ protection against paracetamol induced hepatic damage by reversal of the altered level of serum biochemical parameters and by minimising the histopathological abnormalities. Among all the fractions, percentage protection shown by BNF-LCME at 50 and $100 \mathrm{mg} / \mathrm{kg}$ b.w.p.o. was comparable to that of standard drug, Silymarin $100 \mathrm{mg} / \mathrm{kg}$ b.w.

Table 3 Effect of methanolic extract fractions of LCME on different serum biochemical parameters in paracetamol induced hepatotoxicity in rats

\begin{tabular}{|c|c|c|c|c|c|c|c|c|c|c|c|}
\hline GROUPS & $\begin{array}{l}\text { AST } \\
(\mathrm{U} / \mathrm{l})\end{array}$ & $\begin{array}{l}\mathrm{ALT} \\
(\mathrm{U} / \mathrm{l})\end{array}$ & $\begin{array}{l}\text { ALP } \\
(\mathrm{U} / \mathrm{l})\end{array}$ & $\begin{array}{l}\mathrm{LDH} \\
(\mathrm{U} / \mathrm{l})\end{array}$ & $\begin{array}{l}\mathrm{GGT} \\
(\mathrm{U} / \mathrm{l})\end{array}$ & $\begin{array}{l}\text { TB } \\
(\mathrm{mg} / \mathrm{dl})\end{array}$ & $\begin{array}{l}\mathrm{DB} \\
(\mathrm{mg} / \mathrm{dl})\end{array}$ & $\begin{array}{l}\mathrm{CHOL} \\
(\mathrm{mg} / \mathrm{dl})\end{array}$ & $\begin{array}{l}\text { TP } \\
(g / d l)\end{array}$ & $\begin{array}{l}\text { ALB } \\
(\mathrm{g} / \mathrm{dl})\end{array}$ & $\begin{array}{l}\text { PT } \\
\text { (seconds) }\end{array}$ \\
\hline Normal & $\begin{array}{l}62.18 \pm \\
4.13\end{array}$ & $\begin{array}{l}61.51 \pm \\
5.89\end{array}$ & $\begin{array}{l}442.5 \pm \\
12.31\end{array}$ & $\begin{array}{l}215.61 \pm \\
6.81\end{array}$ & $\begin{array}{l}3.83 \pm \\
0.39\end{array}$ & $\begin{array}{l}0.13 \pm \\
0.003\end{array}$ & $\begin{array}{l}0.04 \pm \\
0.005\end{array}$ & $44.18 \pm 4.84$ & $\begin{array}{l}7.81 \pm \\
0.52\end{array}$ & $\begin{array}{l}3.53 \pm \\
0.32\end{array}$ & $\begin{array}{l}14.16 \pm \\
2.31\end{array}$ \\
\hline Toxic & $\begin{array}{l}145.16 \pm \\
8.18\end{array}$ & $\begin{array}{l}167.35 \pm \\
6.29\end{array}$ & $\begin{array}{l}852.15 \pm \\
16.84\end{array}$ & $\begin{array}{l}363.51 \pm \\
7.16\end{array}$ & $\begin{array}{l}31.38 \pm \\
0.67\end{array}$ & $\begin{array}{l}2.98 \pm \\
0.21\end{array}$ & $\begin{array}{l}1.32 \pm \\
0.05\end{array}$ & $\begin{array}{l}155.31 \pm \\
10.18\end{array}$ & $\begin{array}{l}4.01 \pm \\
0.32\end{array}$ & $\begin{array}{l}1.68 \pm \\
0.14\end{array}$ & $\begin{array}{l}149.19 \pm \\
6.15\end{array}$ \\
\hline $\begin{array}{l}\text { Silymarin } 100 \\
\mathrm{mg} / \mathrm{kg}\end{array}$ & $\begin{array}{l}76.18 \pm \\
1.37^{* * *} \\
(83.7 \%)\end{array}$ & $\begin{array}{l}76.31 \pm \\
4.12^{* * * *} \\
(85.01 \%)\end{array}$ & $\begin{array}{l}502.18 \pm \\
12.4^{* * *} \\
(80.52 \%)\end{array}$ & $\begin{array}{l}240.38 \pm \\
4.93^{* * *} \pm \\
(83 \%)\end{array}$ & $\begin{array}{l}8.78+\frac{ \pm}{* * * *} \\
1.32^{* * *} \\
(82.03 \%)\end{array}$ & $\begin{array}{l}0.59 \pm \\
0.02^{* * *} \\
(83.85 \%)\end{array}$ & $\begin{array}{l}0.27 \underset{ \pm}{ \pm} \\
0.005^{* * *} \\
(82.03 \%)\end{array}$ & $\begin{array}{l}63.31 \pm \\
4.93^{* * * *} \\
(82.78 \%)\end{array}$ & $\begin{array}{l}7.14+\frac{ \pm}{* .5} \\
0.56^{* * *} \\
(82.36 \%)\end{array}$ & $\begin{array}{l}3.21 \pm \\
0.16^{* * * *} \\
(82.7 \%)\end{array}$ & $\begin{array}{l}38.43 \pm \\
3.16^{* * * *} \\
(83.2 \%)\end{array}$ \\
\hline TLF-LCME 50 & $\begin{array}{l}109.3 \pm \\
6.81^{* * *} \\
(43.37 \%)\end{array}$ & $\begin{array}{l}118.31 \pm \\
4.32^{* * *} \pm \\
(46.2 \%)\end{array}$ & $\begin{array}{l}653.25 \pm \\
11.38^{* * * *} \\
(48.5 \%)\end{array}$ & $\begin{array}{l}298.11 \pm \\
4.87^{* * *} \\
(43.9 \%)\end{array}$ & $\begin{array}{l}19.06 \pm \\
2.31^{* * * *} \\
(44.35 \%)\end{array}$ & $\begin{array}{l}1.43^{ \pm \pm} \\
0.04^{* * *} \\
(54.38 \%)\end{array}$ & $\begin{array}{l}0.69 \pm \\
0.02^{* * * *} \\
(50.7 \%)\end{array}$ & $\begin{array}{l}104.39 \pm \\
10.14^{* * *} \\
(45.94 \%)\end{array}$ & $\begin{array}{l}5.83 \pm \\
0.71^{* * *} \\
(47.89 \%)\end{array}$ & $\begin{array}{l}2.45 \pm \\
0.51^{* * *} \\
(41.62 \%)\end{array}$ & $\begin{array}{l}80.41 \pm \\
3.65^{* * *} \\
(51.11 \%)\end{array}$ \\
\hline TLF-LCME 100 & $\begin{array}{l}112.36 \pm \\
7.23^{* * *} \\
(39.75 \%)\end{array}$ & $\begin{array}{l}123.93 \pm \\
6.72^{* * *} \\
(41.5 \%)\end{array}$ & $\begin{array}{l}670.34 \pm \\
12.98^{* * *} \\
(44.3 \%)\end{array}$ & $\begin{array}{l}305.38 \pm \\
7.63^{* * *} \pm \\
(39.1 \%)\end{array}$ & $\begin{array}{l}21.31 \pm \\
3.16^{* * *} \\
(37.05 \%)\end{array}$ & $\begin{array}{l}1.51 \pm \\
0.01^{* * * *} \\
(51.57 \%)\end{array}$ & $\begin{array}{l}0.75 \pm \\
0.05^{* * *} \\
(44.53 \%)\end{array}$ & $\begin{array}{l}111.18 \pm \\
11.3^{* * *} \\
(39.6 \%)\end{array}$ & $\begin{array}{l}5.23 \pm \\
0.81^{* * * *} \\
(32.1 \%)\end{array}$ & $\begin{array}{l}2.32 \pm \\
0.19^{* * *} \\
(34.5 \%)\end{array}$ & $\begin{array}{l}84.31 \pm \\
4.51^{* * * *} \\
(48.14 \%)\end{array}$ \\
\hline BNF-LCME 50 & $\begin{array}{l}79.38 \pm \\
7.19^{* * *} \\
(79.5 \%)\end{array}$ & $\begin{array}{l}82.49 \pm \\
2.28^{* * *} \\
(80.14 \%)\end{array}$ & $\begin{array}{l}529.81 \pm \\
11.34^{* * *} \\
(78.78 \%)\end{array}$ & $\begin{array}{l}247.42 \pm \\
5.31^{* * *} \\
(78.37 \%)\end{array}$ & $\begin{array}{l}9.86 \pm \\
1.16^{* * *} \\
(78.11 \%)\end{array}$ & $\begin{array}{l}0.72 \pm \\
0.02 * * * \\
(79.28 \%)\end{array}$ & $\begin{array}{l}0.30 \pm \\
0.002^{* * *} \\
(79.1 \%)\end{array}$ & $\begin{array}{l}66.31 \pm \\
4.76^{* * *} \\
(79.37 \%)\end{array}$ & $\begin{array}{l}7.01 \pm \\
0.64^{* * *} \\
(78.94 \%)\end{array}$ & $\begin{array}{l}3.18 \pm \\
0.11^{* * *} \\
(79.12 \%)\end{array}$ & $\begin{array}{l}42.25 \pm \\
4.46 * * * \\
(79.25 \%)\end{array}$ \\
\hline BNF-LCME 100 & $\begin{array}{l}85.18 \pm \\
4.34^{* * *} \\
(72.28 \%)\end{array}$ & $\begin{array}{l}85.34 \pm \\
5.45^{* * *} \\
(77.51 \%)\end{array}$ & $\begin{array}{l}550.38 \pm \\
4.81^{* * *} \\
(73.65 \%)\end{array}$ & $\begin{array}{l}256.38 \pm \\
6.18^{* * *} \\
(72.29 \%)\end{array}$ & $\begin{array}{l}10.04 \pm \\
1.38^{* * *} \\
(77.16 \%)\end{array}$ & $\begin{array}{l}0.82 \pm \\
0.05^{* * *} \\
(75.78 \%)\end{array}$ & $\begin{array}{l}0.36 \pm \\
0.05^{* * *} \\
(75 \%)\end{array}$ & $\begin{array}{l}76.34 \pm \\
5.62^{* * * *} \\
(71.11 \%)\end{array}$ & $\begin{array}{l}6.93 \pm \\
0.59^{* * *} \\
(76.84 \%)\end{array}$ & $\begin{array}{l}3.12 \pm \\
0.23^{* * *} \\
(77.83 \%)\end{array}$ & $\begin{array}{l}48.65 \pm \\
3.12^{* * * *} \\
(74.81 \%)\end{array}$ \\
\hline AQF-LCME 50 & $\begin{array}{l}98.16 \pm \\
5.74^{* * *} \\
(56.6 \%)\end{array}$ & $\begin{array}{l}102.27 \pm \\
6.63^{* * *} \\
(61.2 \%)\end{array}$ & $\begin{array}{l}609.1 \pm \\
12.19^{* * *} \\
(59.2 \%)\end{array}$ & $\begin{array}{l}280.29 \pm \\
5.18^{* * *} \\
(56.08 \%)\end{array}$ & $\begin{array}{l}16.31 \pm \\
2.51^{* * * *} \\
(54.45 \%)\end{array}$ & $\begin{array}{l}1.41 \pm \\
0.03^{* * *} \\
(55.08 \%)\end{array}$ & $\begin{array}{l}0.51 \pm \\
0.05^{* * *} \\
(58.59 \%)\end{array}$ & $\begin{array}{l}93.74 \pm \\
4.83^{* * *} \\
(55.85 \%)\end{array}$ & $\begin{array}{l}6.21 \pm \\
0.53^{* * *} \\
(57.36 \%)\end{array}$ & $\begin{array}{l}2.76 \pm \\
0.51^{* * *} \\
(58.37 \%)\end{array}$ & $\begin{array}{l}72.43 \pm \\
6.31^{* * *} \\
(57.03 \%)\end{array}$ \\
\hline AQF-LCME 100 & $\begin{array}{l}103.33 \pm \\
4.38^{* * *} \\
(50.6 \%)\end{array}$ & $\begin{array}{l}109.43 \pm \\
5.49^{* * *} \\
(54.71 \%)\end{array}$ & $\begin{array}{l}622.16 \pm \\
11.47^{* * *} \\
(56.09 \%)\end{array}$ & $\begin{array}{l}287.53 \pm \\
6.17^{* * *} \\
(51.35 \%)\end{array}$ & $\begin{array}{l}17.18 \pm \\
3.36^{* * *} \\
(51.55 \%)\end{array}$ & $\begin{array}{l}1.49 \pm \\
0.03^{* * * *} \\
(52.2 \%)\end{array}$ & $\begin{array}{l}0.59 \pm \\
0.003^{* * *} \\
(56.13 \%)\end{array}$ & $\begin{array}{l}98.18 \pm \\
3.36^{* * * *} \\
(51.32 \%)\end{array}$ & $\begin{array}{l}6.09 \pm \\
0.11^{* * * *} \\
(52.1 \%)\end{array}$ & $\begin{array}{l}2.55 \pm \\
0.41^{* * *} \\
(47.02 \%)\end{array}$ & $\begin{array}{l}79.31 \pm \\
5.56^{* * *} \\
(51.85 \%)\end{array}$ \\
\hline
\end{tabular}

Data expressed as mean $\pm \mathrm{SD}, n=6$, values in parenthesis indicate percentage recovery

TLF-LCME Toulene fraction of methanolic extract fraction of Lindernia ciliata, BNF-LCME Butanone fraction of methanolic extract fraction of Lindernia ciliata, AQFLCME Aqueous fraction of methanolic extract fraction of Lindernia ciliata

$p$ value-paracetamol Vs vehicle; $p$ value paracetamol Vs treatments- ${ }^{*}<0.05 ;^{* *}<0.01 ;{ }^{* * *}<0.001$ 


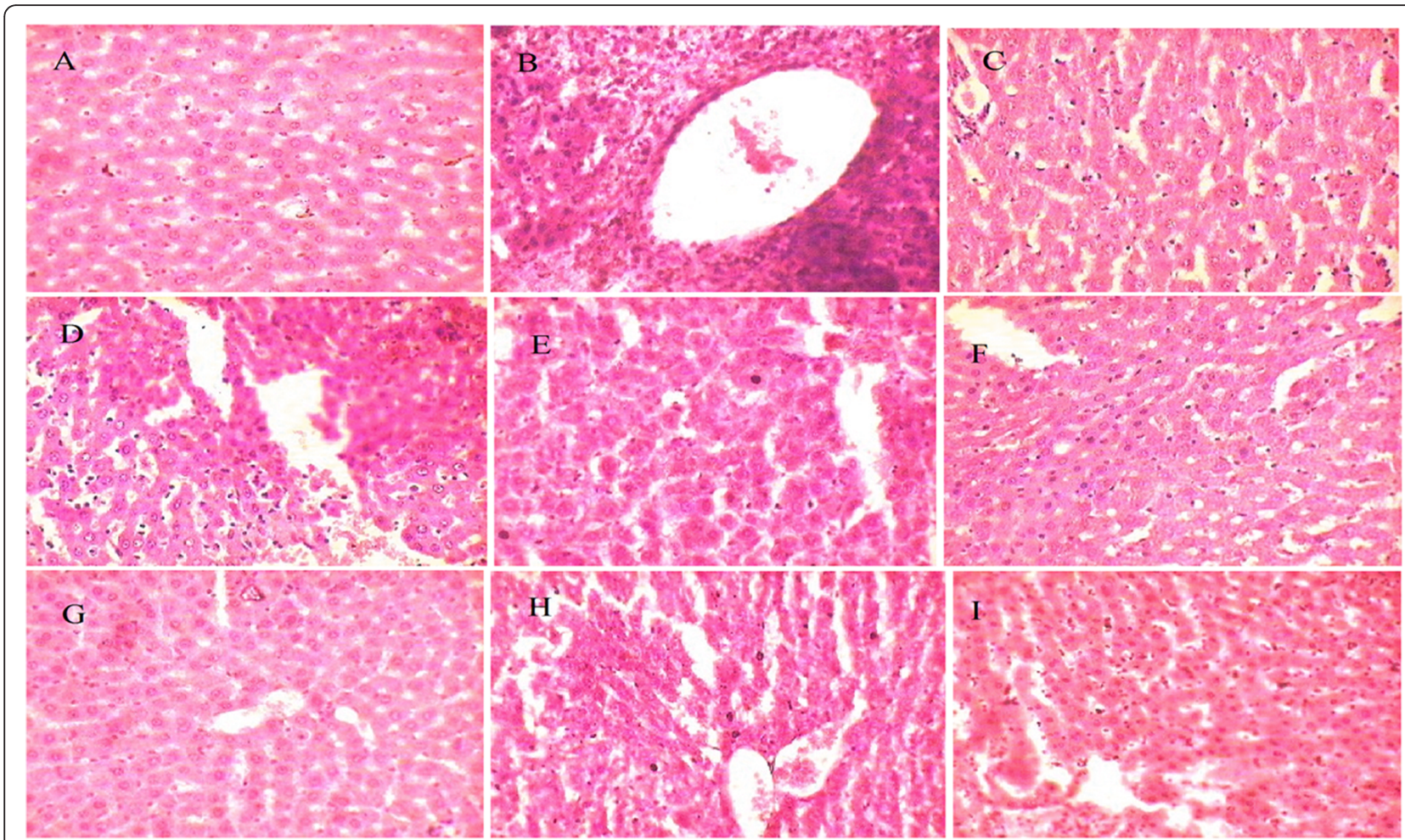

Fig. 1 Effect of fractions of LCME on histopathological changes in liver of rats in paracetamol induced hepatotoxicity in rats. Done by using Digital Motic Microscope, Magification 100X. a = Normal, $\mathbf{b}=$ Toxic, $\mathbf{c}=$ Standard, $\mathbf{d}=$ TLF-LCME 50, e = TLF-LCME 100, $\mathbf{f}=B N F-L C M E$ 50, $\mathbf{g}=$ BNFLCME 100, h = AQF-LCME 50, i = AQF-LCME 100, TLF-LCME: Toulene fraction of methanolic extract fraction of Lindernia ciliata; BNF-LCME: Butanol fraction of methanolic extract fraction of Lindernia ciliata; AQF-LCME: Aqueous fraction of methanolic extract fraction of Lindernia ciliata

\section{Assessment of antihepatotoxic activity of BNF-LCME against $\mathrm{D}$-Galactosamine induced hepatotoxicity in rats} Among all the fractions, BNF-LCME at both test doses has shown remarkable protection against paracetamol induced hepatic damage in rats. Hence the same is selected to assess the curative effect against D-Galactosamine induced hepatotoxicity in rats. The results of the study indicating serum biochemical parameters and prothrombin time are shown in Table 4 whereas the results of in vivo antioxidant activity are presented in Table 5 and histological results are

Table 4 Effect of BNF-LCME on different serum biochemical parameters and prothrombin time in D-galactosamine induced hepatotoxicity in rats

\begin{tabular}{|c|c|c|c|c|c|c|c|c|c|c|}
\hline Groups & $\begin{array}{l}\text { Glucose } \\
(\mathrm{U} / \mathrm{L})\end{array}$ & $\begin{array}{l}\text { AST } \\
(U / L)\end{array}$ & $\begin{array}{l}\mathrm{ALT} \\
(\mathrm{U} / \mathrm{L})\end{array}$ & $\begin{array}{l}\text { ALP } \\
(U / L)\end{array}$ & $\begin{array}{l}\text { TB } \\
(\mathrm{mg} / \mathrm{dL})\end{array}$ & $\begin{array}{l}\mathrm{DB} \\
(\mathrm{mg} / \mathrm{dL})\end{array}$ & $\begin{array}{l}\text { TP } \\
(\mathrm{g} / \mathrm{dL})\end{array}$ & $\begin{array}{l}\text { ALB } \\
(g / d L)\end{array}$ & $\begin{array}{l}\mathrm{LDH} \\
(\mathrm{U} / \mathrm{L})\end{array}$ & $\begin{array}{l}\text { PT } \\
\text { (seconds) }\end{array}$ \\
\hline Normal & $91.6 \pm 3.3$ & $60.3 \pm 3.1$ & $58.1 \pm 4.3$ & $523 \pm 9.71$ & $0.19 \pm 0.05$ & $0.04 \pm 0.005$ & $7.91 \pm 0.31$ & $3.86 \pm 0.22$ & $212.73 \pm 6.72$ & $14.35 \pm 2.15$ \\
\hline Toxic & $48 . .3 \pm 1.9$ & $\begin{array}{l}241.4 \pm \\
3.7\end{array}$ & $\begin{array}{l}186.3 \pm \\
5.1\end{array}$ & $967.3 \pm 10.3$ & $2.81 \pm 0.13$ & $1.14 \pm 0.08$ & $4.61 \pm 0.44$ & $2.01 \pm 0.12$ & $396.11 \pm 8.13$ & $162.21 \pm 3.4$ \\
\hline $\begin{array}{l}\text { Silymarin } 100 \\
\mathrm{mg} / \mathrm{kg}\end{array}$ & $\begin{array}{l}84.6 \pm \\
3.5^{* * *} \\
83.83 \%\end{array}$ & $\begin{array}{l}89.1 \pm \\
5.6^{* * *} \\
84.09 \%\end{array}$ & $\begin{array}{l}78.1 \pm \\
3.6^{* * *} \\
84.33 \%\end{array}$ & $\begin{array}{l}602.1 \pm \\
6.78^{* * * *} \\
82.12 \%\end{array}$ & $\begin{array}{l}0.62 \pm \\
0.005^{* * *} \\
83.58 \%\end{array}$ & $\begin{array}{l}0.22 \pm^{*^{* *}} \\
0.005^{*} \\
86.63 \%\end{array}$ & $\begin{array}{l}7.25 \pm \\
0.13^{* * * *} \\
80.78 \%\end{array}$ & $\begin{array}{l}3.465 \pm \\
0.55^{* * *} \\
78.36 \%\end{array}$ & $\begin{array}{l}241.33 \pm \\
7.61^{* * *} \\
83.82 \%\end{array}$ & $\begin{array}{l}38.31 \pm \\
2.64^{* * * *} \\
83.4 \%\end{array}$ \\
\hline BNF-LCME 50 & $\begin{array}{l}77.5 \pm \\
1.8^{* * * *} \\
67.45 \%\end{array}$ & $\begin{array}{l}93.6 \pm \\
1.9^{* * *} \\
81.69 \%\end{array}$ & $\begin{array}{l}82.3 \pm \\
4.8^{* * * *} \\
81.12 \%\end{array}$ & $\begin{array}{l}623 \underset{ \pm * *}{ \pm} \\
7.31^{* *} \\
77.22 \%\end{array}$ & $\begin{array}{l}0.68 \pm \\
0.04^{* * * *} \\
81.29 \%\end{array}$ & $\begin{array}{l}0.26 \pm \\
0.06^{* * * *} \\
80.01 \%\end{array}$ & $\begin{array}{l}7.19 \pm \\
0.21^{* * * *} \\
78.81 \%\end{array}$ & $\begin{array}{l}3.38 \pm \\
0.19^{* * * *} \\
74.35 \%\end{array}$ & $\begin{array}{l}251.64 \pm \\
6.35^{* * *} \\
78.80 \%\end{array}$ & $\begin{array}{l}41.45 \pm \\
2.86^{* * * *} \\
81.6 \%\end{array}$ \\
\hline BNF-LCME 100 & $\begin{array}{l}75.3 \pm \\
3.6^{* * *} \\
62.5 \%\end{array}$ & $\begin{array}{l}99.1 \pm \\
3.2^{* * *} \\
78.57 \%\end{array}$ & $\begin{array}{l}89.3 \pm \\
7.6^{* * *} \\
75.66 \%\end{array}$ & $\begin{array}{l}637.1 \pm \\
8.91^{* * *} \\
74.31 \%\end{array}$ & $\begin{array}{l}0.76 \pm \\
0.05^{* * *} \\
78.24 \%\end{array}$ & $\begin{array}{l}0.29 \pm \\
0.001^{* * *} \\
77.27 \%\end{array}$ & $\begin{array}{l}6.93 \pm \\
0.37^{* * * *} \\
70.3 \%\end{array}$ & $\begin{array}{l}3.16 \pm \\
0.25^{* * *} \\
62.5\end{array}$ & $\begin{array}{l}259.38 \pm \\
6.5^{* * *} \\
74.2\end{array}$ & $\begin{array}{l}48.34 \pm \\
2.96^{* * * *} \\
77.01\end{array}$ \\
\hline
\end{tabular}

Data expressed as mean $\pm S D, n=6$, values in parenthesis indicate percentage recovery

$p$ value- D-galactosamine Vs vehicle; $p$ value D-galactosamine Vs treatments- ${ }^{*}<0.05 ;{ }^{* *}<0.01 i^{* * *}<0.001$

BNF-LCME Butanone fraction of methanolic extract of Lindernia ciliata 
Table 5 The effect of BNF-LCME on in vivo antioxidant parameters against D-Galactosamine induced hepatic damage in rats

\begin{tabular}{llll}
\hline Groups & $\begin{array}{l}\text { MDA } \\
(\mathrm{mM} / \mathrm{mg})\end{array}$ & $\begin{array}{l}\mathrm{GSH} \\
(\mathrm{nM} / \mathrm{mg})\end{array}$ & $\begin{array}{l}\text { CAT } \\
(\mathrm{U} / \mathrm{mg})\end{array}$ \\
\hline Normal & $1.44 \pm 0.03$ & $5.96 \pm 0.190$ & $10.36 \pm 0.18$ \\
Toxic & $3.92 \pm 0.06$ & $1.4 \pm 0.03$ & $3.41 \pm 0.09$ \\
Silymarin 100 mg/kg & $1.92 \pm 0.04$ & $5.11 \pm 0.23$ & $8.96 \pm 0.11$ \\
& $(80.64 \%)$ & $(81.35 \%)$ & $(79.85 \%)$ \\
BNF-LCME 50 & $2.11 \pm 0.16$ & $4.65 \pm 0.08$ & $8.51 \pm 0.56$ \\
& $(72.98 \%)$ & $(71.21 \%)$ & $(73.38 \%)$ \\
BNF-LCME 100 & $2.31 \pm 0.14$ & $4.54 \pm 0.11$ & $8.19 \pm 0.16$ \\
& $(64.14 \%)$ & $(68.82 \%)$ & $(69.21 \%)$ \\
\hline
\end{tabular}

Data expressed as mean $\pm S D, n=6$, values in parenthesis indicate percentage recovery

$p$ value- D-Galactosamine Vs vehicle; $p$ value D-Galactosamine Vs treatments$"<0.05 ; i^{* *}<0.01 i^{* * *}<0.001$

BNF-LCME Butanone fraction of methanolic extract of Lindernia ciliata

shown in Fig. 2. The hepatotoxicity induced by D-Galactosamine (D-Galactosamine) resembles that of human viral hepatitis both in metabolic and morphological aberrations that always caused peri-portal inflammation [14] and hepatocyte apoptosis [15]. Treatment with BNFLCME at 50 and $100 \mathrm{mg} / \mathrm{kg}$ decreased the level of serum ALT, AST, ALP and LDH, suggesting extract's ability to scavenge reactive oxygen species generated from DGalactosamine intoxication and hence prevent hepatic cellular enzymes from leaking into the blood. Increase in serum bilirubin level in D-Galactosamine treated rats is due to abnormal excretion of bile by the liver. Administration of BNF-LCME at 50 and $100 \mathrm{mg} / \mathrm{kg}$ decreased the serum bilirubin level indicating the extract's ability to repair the damaged hepatocytes. In addition it also increased the reduced levels of TP and ALB in serum which may be attributed to the extract's ability to stabilize endoplasmic reticulum and trigger protein synthesis. The extract also increased the serum GLU level by eliminating the toxic metabolite and accelerating the formation of hepatic UTP. Liver synthesizes different clotting factors such as I, II, V, VII, IX and X [16]. Apart from the effect on other hepatospecific parameters, the fraction, BNF-LCME also showed a significant $(p<0.001)$ recovery in $\mathrm{PT}$ which indicates the improved synthetic capacity of the liver.

The histological examination of the liver of rats treated with D-Galactosamine showed inflammation of portal tract, hyperplasia of kupffer cells, vacuolization of hepatocytes and bleeding in midzonal areas. Treatment with BNF-LCME at 50 and $100 \mathrm{mg} / \mathrm{kg}$ has shown significant recovery against the damage, which may be due to prevention of accumulation of UDPGalactosamine in liver cells there by inhibiting various signaling pathways leading to apoptotic cell death.

The increase in MDA or decrease in GSH and CAT levels in liver homogenate of D-Galactosamine treated rats indicates the lipid peroxidation. MDA is one of

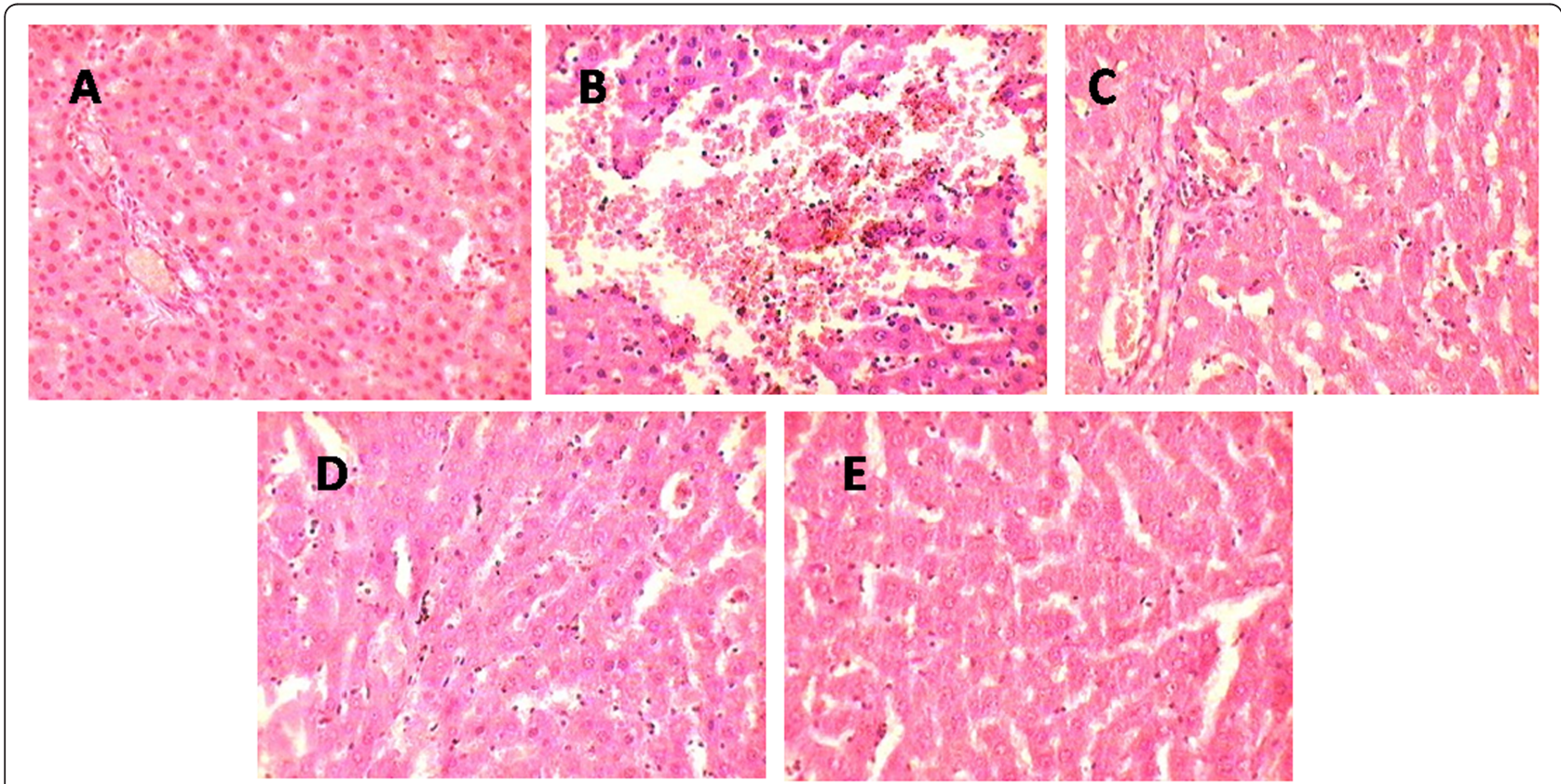

Fig. 2 Antihepatotoxic activity of BNF-LCME against D-Galactosamine induced hepatotoxicity in rats. Done by using Digital Motic Microscope, Magification 100X. a: normal, b: Toxic, c: Standard, d:BNF-LCME-50, e:BNF-LCME-100, BNF-LCME: Butanone fraction of methanolic extract fraction of Lindernia ciliata 
the end products resulting from the peroxidation of biological membrane composing of polyunsaturated fatty acids (PUFA) [17]. Glutathione (GSH) is a non enzymatic antioxidant which prevents damage to important cellular components caused by reactive oxygen species such as free radicals and peroxides [18]. CAT is known to breakdown $\mathrm{H}_{2} \mathrm{O}_{2}$ to $\mathrm{H}_{2} \mathrm{O}$ and $\mathrm{O}_{2}$ and can be found in the peroxisome and mitochondria, especially in liver. The groups receivedBNFLCME and Standard prior to D-Galactosamine administration exhibited significant protection against lipid peroxidation as evident from decrease in MDA level increase in GSH and CAT levels indicating the in vivo antioxidant potential of BNF-LCME. The significant antioxidant effect of BNF-LCME revealed that it has the ability to ameliorate oxidative stress and preserve hepatic function against free radicals produced by D-Galactosamine intoxification.

\section{High performance liquid chromatography of BNF-LCME}

Plant extracts are complex mixtures of varied chemicals, which pose a problem in standardization and quality control, but these chemicals are responsible for imparting therapeutic effect with the advantage of synergistic and additive effects and at the same time having fewer side effects [19]. Consequently, the herbal drug preparation itself as a whole is regarded as the active substance. Hence, the reproducibility of the
Table 6 The HPLC chromatogram of BNF-LCME

\begin{tabular}{lllll}
\hline Sample ID & Name & Ret. Time & Area & \% Area \\
\hline 1 & Unknown & 1.848 & 727,295 & 34.82 \\
2 & Unknown & 2.549 & 821,857 & 39.35 \\
3 & Unknown & 3.846 & 539,388 & 25.82 \\
\hline
\end{tabular}

total configuration of herbal drug constituents is important. To meet this requirement it is essential to establish the chemo profiles of the samples by TLC/ HPLC/GLC/ HPTLC. Hence, TLC and HPLC profile of the selected bio active fraction, BNF-LCME was developed to ensure its quality.

The results of the HPLC study are shown in Fig. 3 and Table 6. The HPLC chromatogram of BNFLCME showed three peaks corresponding to at least 3 compounds. Among the three peaks, two peaks were found to be major with percentage peak area $34.82 \%$ and $39.35 \%$ at retention times 1.848 and 2.54 min respectively.

\section{Conclusions}

All the fractions of LCME exhibited significant hepatoprotective activity and BNF-LCME $(50 \mathrm{mg} / \mathrm{kg}$ ) was identified as the most effective fraction which may be attributed to the flavonoidal/phenolic compounds present in the fraction.

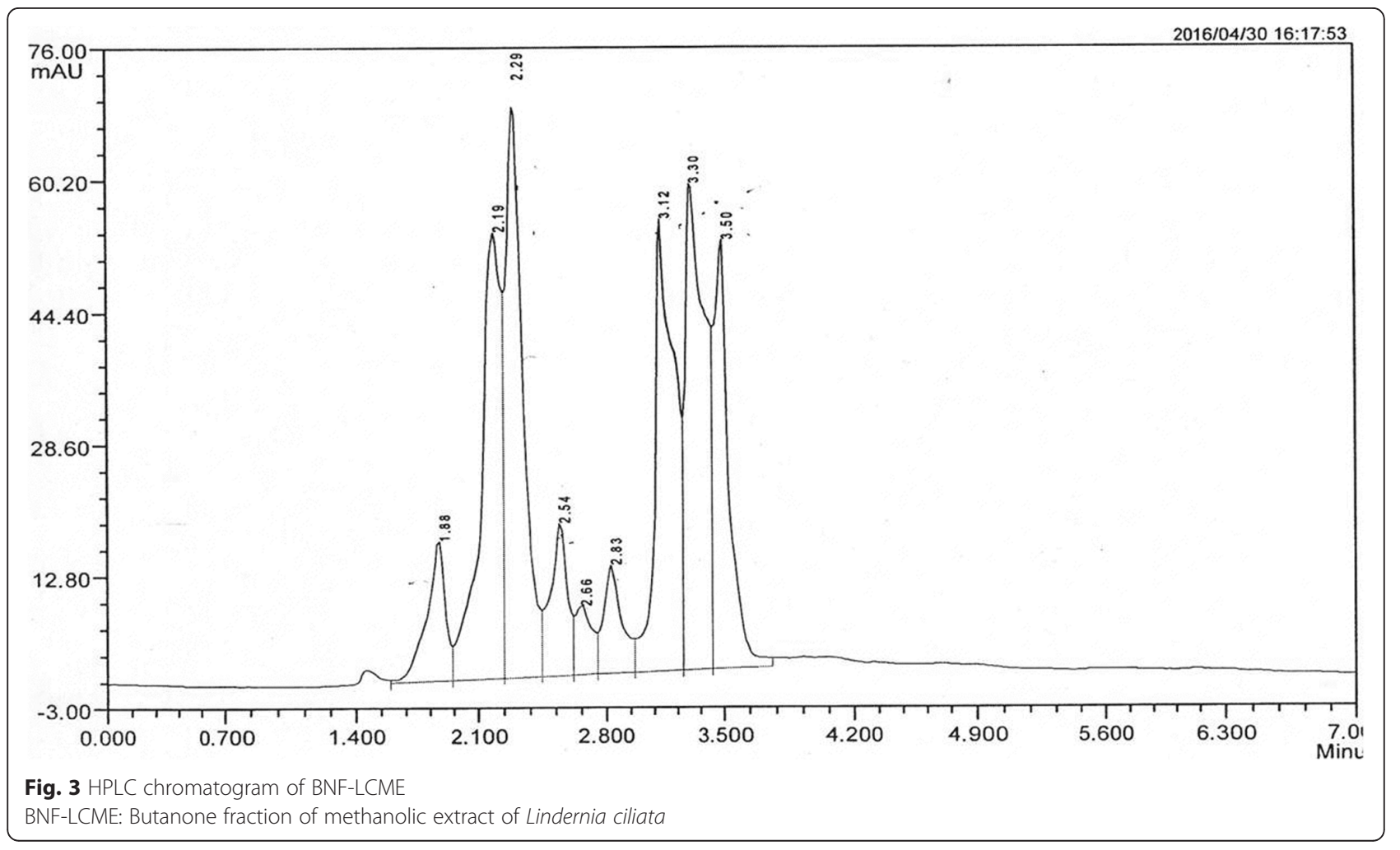




\section{Abbreviations}

AAE: Ascorbic acid equivalent; ALB: Albumin; ALP: Alkaline phosphatase; ALT: Alanine transaminase; AQF-LCME: Aqueous fraction of LCME; AST: Aspartate transaminase; BNF-LCME: Butanone fraction of LCME; CAT: Catalase; DB: Direct bilirubin; DPPH: 1,1-Diphenyl-2-picrylhydrazyl radical; GAE: Gallic acid equivalents; GGT: Gamma glutamyl transferase; GSH: Glutathione; LCME: Methanolic extract of Lindernia ciliata; LDH: Lactate dehydrogenase; MDA: Melondialdehyde; RE: Rutin equivalents; TB: Total bilirubin; TLF-LCME: Toluene fraction of LCME; TP: Total protein

\section{Acknowledgements}

Not applicable

\section{Authors' contributions}

PP performed all the animal studies, analyzed and interpreted the data and was a major contributor in writing the manuscript. NRY performed the histological examination of the liver, and in vivo antioxidant studies. RKB performed the phytochemical studies, selection, collection and preparation of plant extract fractions. All authors read and approved the final manuscript.

\section{Authors' information}

BRK is Dr. B. Ravi Kumar, Professor, in University college of Pharmaceutical sciences, Kakatiya University, Warangal, Telangana, India with 30 years of research experience.

YNR is Dr. Yellu Narsimha Reddy, Professor, in University college of

Pharmaceutical sciences, Kakatiya University, Warangal, Telangana, India with 25 years of research experience.

PP is Dr. P. Praneetha, pursued PhD under the joint supervision of Prof. BRK and Prof.YNR, in University college of Pharmaceutical sciences, Kakatiya University, Warangal, Telangana, India.

\section{Funding}

This work was supported by a grant (UGC sanction No.F.7-106/2007 (BSR), dated January 16, 2012) from UGC-BSR Meritorious Research Fellowship, New Delhi.

\section{Availability of data and materials}

All the data was analysed by using the software, Graph pad prism 3.0 version which is available online.

\section{Ethics approval and consent to participate}

The animal studies were performed with a prior permission from our institutional animal ethical committee with registered number.

\section{Consent for publication}

Not applicable

\section{Competing interests}

The authors declare that they have no competing interests.

Received: 4 June 2019 Accepted: 23 August 2019

Published online: 01 October 2019

\section{References}

1. Neamsuvan O, Singdam P, Yingcharoen K, Sengnon N. A survey of medicinal plants in mangrove and beach forests from sating Phra peninsula, Songkhla Province, Thailand. J Med Plant Res. 2012;6:2421-37.

2. Devi CB, Singh NKS, Ram SS, Sudarshan M, Chakraborty A, Rajmuhon N. Trace element profile of some selected medicinal plants of Manipur, India. Sci J Pure Appl Sci. 2013;2:332-40.

3. Praneetha P, Narsimha Reddy Y, Ravi Kumar B. In-vitro and In-vivo hepatoprotective studies on methanolic extract of aerial parts of Ludwigia hyssopifolia G Don Exell. Pharmacogn Mag. 2019;14:546-55.

4. Blios MS. Antioxidant determination by the use of stable free radical. Nature. 1958;181:1199-200.

5. Jiang C, Jiao Y, Chen X, Li X, Yan W, Yu B, Xiong Q. Preliminary characterization and potential hepatoprotective effect of polysaccharides from Cipangopaludina chinensis. Food Chem Toxicol. 2013;59:18-25.

6. Snezana MM, Aleksandar ND, Nebojsa LA, Silvana AA, Tatjana SK, Gordana MB, Mirjana BVM, Radmila ZK. Nitric oxide-scavenging activity of polyhydroxylated fullerenol, $\mathrm{C}_{6} \mathrm{O}(\mathrm{OH})_{24}$. Nitric Oxide. 2004;11:201-7.
7. Hazra B, Biswas S, Mandal N. Antioxidant and free radical scavenging activity of Spondias pinnata. BMC Complement Altern Med. 2008:8:63.

8. Bursal E, Koksal E. Evaluation of reducing power and radical scavenging activities of water and ethanol extracts from sumac (Rhus coriaria L.). Food Res Int. 2011:44:2217-21.

9. OECD guidelines for testing of chemicals (India). Acute oral toxicity Test No423, India. 2001. https://www.oecd.org/chemicalsafety/risk-assessment/194 8378.pdf. Accessed 17 Dec 2001.

10. Sabeena S, Ajay GN. Hepatoprotective effect of leaves of Erythroxylum monogynum Roxb. On paracetamol induced toxicity. Asian Pac J Trop Biomed. 2013:3(Suppl 11):877-81.

11. Karan M, Vasisht K, Handa SS. Antihepatotoxic activity of Swertia chirata against paracetamol and galactosamine induced hepatotoxicity in rats. Phytother Res. 1999;13:95-101.

12. Glover RP, Kuzell WC. Prothrombin time-determination by a whole blood micro-method for control of anticoagulant therapy. Calif Med. 1961; 95(Suppl 1):24-9.

13. Praneetha $P$, Narsimha Reddy $Y$, Ravi Kumar B. In-vitro and in-vivo Hepatoprotective studies on methanolic extract of aerial parts of Ludwigia hyssopifolia G. Don Exell Pharmacog Mag. 2019;14:546-55.

14. Keppler D, Lesch R, Reutter W, Decker K. Experimental hepatitis induced by D-galactosamine. Exp Mol Pathol. 1968:9:279-90.

15. Decker K, Keppler D. Galactosamine hepatitis: key role of the nucleotide deficiency period in the pathogenesis of cell injury and cell death. Rev Physiol Biochem Pharmacol. 1974;71:77-106.

16. Suttie JW, Jackson CM. Prothrombin structure, activation, and biosynthesis. Physiol Rev. 1977;57:1-70.

17. Vaca CE, Wilhelm J, Harms-Rihsdahl M. Interaction of lipid peroxidation product with DNA. A review. Mutat Res-Rev Mutat. 1988;195:137-49.

18. Henry JF, Hongqiao Z, Alessandra R. Glutathione: overview of its protective roles, measurement, and biosynthesis. Mol Asp Med. 2009;30:1-12.

19. Rajani M, Niranjan S, Kanaki NS. Phytochemical standardization of herbal drugs and poly herbal formulations. In: Ramawat KG, Merillon JM, editors. Bioactive molecules and medicinal plants. India: Springer; 2008. p. 349-69.

\section{Publisher's Note}

Springer Nature remains neutral with regard to jurisdictional claims in published maps and institutional affiliations.

\section{Submit your manuscript to a SpringerOpen ${ }^{\circ}$ journal and benefit from:}

- Convenient online submission

- Rigorous peer review

- Open access: articles freely available online

- High visibility within the field

- Retaining the copyright to your article

Submit your next manuscript at $\boldsymbol{\nabla}$ springeropen.com 